\title{
Novel Coronavirus (COVID-19) Outbreak In Nigeria: How Prepared Is The Radiography Sector?
}

\author{
G. M. Akpaniwo, D. A. Olushola, M. C. Okeji, N. O. Egbe
}

\begin{abstract}
Introduction: Following the outbreak and increasing numbers of coronavirus (COVID-19) cases in Nigeria, healthcare professionals are at the forefront in dealing with the pandemic. Radiographers are among the first line care givers. The study is aim to assess the level of preparedness of the Radiography sector in Nigeria.

Methods: A prospective cross-sectional survey was conducted using self-completion questionnaire. The questionnaire was generated on the 26th of March 2020, using online Google forms consisting of closed and open ended questions. Paired t-test statistic was used to compare the difference between "Before and After" changes in departmental protocols, using the SPSS statistical software, version 20 . Statistically significant level was set at 0.05 .

Results: A total of 107 radiographers from across the country responded to the questionnaire. Of these, $78(72 \%)$ were male and $27.1 \%$ female, giving a male to female ratio of 3:1. Following the outbreak of COVID-19, 86\% of the respondents report that there have been changes in the departmental procedures. Sixtyseven percent said "No" to the availability of an appointment system, guidelines to reduce human to human contact(57\%) and auditing for suspected COVID-19 cases (58.9\%). Only 16(15\%) of the respondents had received emergency training towards the fight against COVID-19. Of these, $6(37.5 \%)$ were trained in patient care, only $1(6.3 \%)$ on emergency response while 9 $(56.3 \%)$ received training in limiting human to human transmission.
\end{abstract}

Conclusion: Findings in this study, suggest inadequate preparedness, with evidence of inadequate provision of equipment (Mobile X-ray and Ultrasound machines) for the diagnosis and management of cases in departments and isolation centers. In addition, emergency training on COVID-19 issues is low. The foregoing calls for adoption of urgent and immediate remedial measures.

Implications for practice: This study identify areas of practice that may detract from achieving optimum service delivery and safety during the pandemic.

Index Terms - Novel coronavirus, COVID-19, SARS-CoV-2, Acute respiratory infection, Radiography.

\section{INTRODUCTION}

The coronavirus outbreak, also known as COVID-19, was first reported in December 2019 in the city of Wuhan, Hubei province, located in the central part of China. Wuhan has a population of approximately 11.9 million people [1]-[3]. The COVID-19 is a rare respiratory disease which can be deadly due to massive alveolar damage and progressive respiratory failure [4]-[6]. It was thus declared a global pandemic by the World health organization (WHO). As at April 30, 2020; more than 3,209,984 confirmed cases, 985,957 recovered cases and 228,057 deaths had been reported worldwide [7], while in Nigeria, a total of 1,728 confirmed cases, 307 recovered cases and 51 deaths were reported at the time of carrying out this study[8].

The novel coronavirus was found to be caused by SARSCoV-2 [3] and has been shown to have phylogenetic similarities as well as pattern of manifestation like that of the severe acute respiratory syndrome (SARS) caused by SARSCoV-1 [9]. In an earlier research conducted in China, in January 2020 by Bastola et al., [10], it was reported that among 41 patients that were confirmed positive to the COVID-19, half of them had underlying diseases such diabetes (20\%), cardiovascular disease (15\%), and hypertension $(15 \%)$. Their symptoms were mainly fever (98\%), cough $(76 \%)$, and fatigue $(44 \%)$. Other severe complications included respiratory distress syndrome (29\%), RNAaemia (15\%), acute cardiac injury (12\%), and other secondary infections. Of the total infected patients, $32 \%$ were admitted to an Intensive Care Unit (ICU) and the death rate was $15 \%$ [10].

Within this period, it was rapidly spread to other regions of china and neighboring countries such as Singapore, Thailand, South Korea, Japan, and Malaysia [11]. The western countries were not left out as it spread to the United State of America, United Kingdom, Spain, and Italy. Currently 220 countries worldwide have been affected as at the time of undertaking this study. Africa's first confirmed case of COVID-19 was reported in Egypt [12], [13]. while Nigeria confirmed her index case on the $27^{\text {th }}$ of February 2020 [14], [15].

Due to its high transmissivity and the current lack of a treatment protocol, the pandemic has altered the dynamics of human living. As of May 20th, 2020; more than 4,897,567 confirmed cases, 1,688,630 recovered cases and 323,286 deaths reported worldwide [7], while in Nigeria, a total of 6401 confirmed cases, 1734 recovered cases and 192 deaths was reported at the time of carrying out this research study[8]

With increasing numbers of coronavirus (COVID-19) cases in Nigeria, it was necessary to examine the degree of preparedness of the different sectors of her healthcare system. The Radiography or Medical Imaging Science sector, one of the professions at the forefront in dealing with the pandemic, is the focus of this study. Radiographers are the first line care givers that facilitate the use of diagnostic Chest X-ray images, Computed Tomography (CT) scanograms and Ultrasound
Published on July 11, 2020.

G. M. Akpaniwo, Usmanu Danfodiyo University Sokoto, Nigeria. (corresponding e-mail: akpaniwo@gmail.com).

D. A. Olushola, Mercy University Hospital, Cork, Republic of Ireland. (e-mail: Higherground200@yahoo.com).
M. C. Okeji, University of Nigeria, Nigeria. (e-mail: mark.okeji@unn.edu.ng).

N. O. Egbe, University of Calabar, Nigeria. (e-mail: nneoyiegbe@gmail.com). 
scans for the diagnosis of, and guidance for the management of the patients' conditions.

Radiological imaging in the diagnosis of COVID-19 involves the acquisition of a Posterior-anterior chest radiograph (PA-CXR). The extent of the COVID-19 infection on CXR is assessed in 3 zones: upper (above the carina), middle (upper half of the craniocaudal distance of the remaining lung), and lower (lower half of the craniocaudal distance of the remaining lung) lung zones. Also, the parenchymal abnormality on CXR is graded on a 3-point scale: 1 , normal attenuation; 2 , ground-glass attenuation; and 3 , consolidation. The ground-glass opacity is an area of hazy increased lung opacity, within which margins of pulmonary vessels may be indistinct [16]. Consolidation appears as a homogeneous increase in pulmonary parenchymal attenuation that obscures the margins of vessels and airway walls [16]. Furthermore, using CT images, the radiological findings summarized by Song et al., [17] and Michael et al.,[18] were ground-glass opacity (GGO; 77\%) followed by GGO with reticular and/or interlobular septal thickening $(75 \%)$ [17], [18]. As the principal workforce in the generation of the diagnostic images, radiographers are at high risk of acquiring and transmitting the infection due to their close contact with patients, and patient's contact with imaging equipment and accessories. As a result of this, it becomes necessary to evaluate what changes have taken place as a measure of how prepared radiographers and the Radiography sector is, in the face of the COVID-19 outbreak in Nigeria.

\section{METHODS}

A prospective, cross-sectional survey was conducted using a self-completion questionnaires. The questionnaire was generated on the 26th of March 2020, using online Google forms consisting of closed and open ended questions. The link to the questionnaire was distributed among radiographers across public and private health sectors in Nigeria, via WhatsApp messenger using radiographer contacts and radiographers chats groups. The link was further re-shared among radiographer colleagues who received the link to the questionnaire and only radiographers who wish to participate in the research study completed and submitted the questionnaire successfully. The questionnaire was designed to evaluate socio-demographics, pre and within covid-19 departmental protocols and guidelines, availability and use of personal protective equipment, protection of radiographic equipment, emergency training and isolation centers toward COVID-19 Outbreak. After a period of one month, the data from the returned questionnaires was analyzed on the 30th of April 2020. The results were presented in simple ratios or proportions, and deductions made there from. Paired t-test was used to compare the difference between "Before and After" changes in departmental protocol, using SPSS version 20. Statistical significant level was set at P-value of $<0.05$.

\section{A. Ethical approval}

Ethical approval was applied for and obtained from Research Ethics Committee of the Radiography Department, Usmanu Danfodiyo University Sokoto.

\section{RESULT}

Of the 107 radiographers that responded, $78(72.9 \%)$ were males and $27.1 \%$ were females, giving a male to female ratio of $3: 1$.

\section{A. Socio-demographics}

The educational qualification of the respondents was distributed as follows; $88(82.2 \%)$ had a B.Sc or B. Rad degrees, $15(14 \%)$ were M.Sc degree holders, 3(2.8\%) had PhD degrees and 1(1\%) held Diploma in Radiography. The respondents had practiced as radiographers for 1-5 years (62.1\%), 6-10 years (27.6\%) and 11-20 years (10.3\%), respectively. Among the respondents, $91 \%$ were Clinical Radiographers, 7.7\% were Academic radiographers and 1.3\% plied their trade in Administrative positions in the health facilities. At least 44 (40.2\%) of the radiographers practiced in Tertiary or teaching hospitals, while $43(41 \%)$ were drawn from private hospitals as shown in Fig. 1.

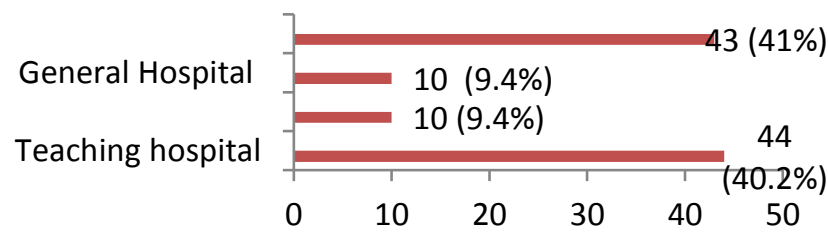

Fig. 1. Distribution of respondent place of work.

\section{B. Departmental protocols and guidelines}

Since the outbreak of COVID-19, $86 \%$ of the respondents agreed that there have been changes in the departmental procedures, However, majority said "No" to the availability of appointment system (67.3\%), guidelines to reduce human to human contact (57\%), and auditing for suspected COVID19 cases $(58.9 \%)$ as shown on Fig. 2.

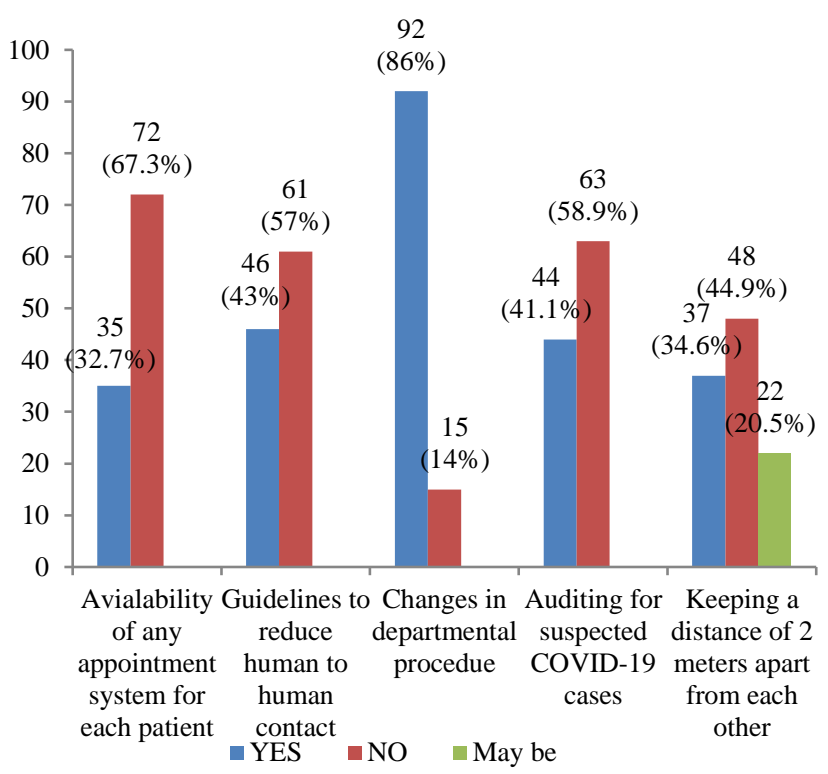

Fig. 2. Response to departmental protocols and guidelines.

\section{Availability and use of personal protective equipment (PPE)}

The participants response to questions on the operational protocol BEFORE the outbreak of COVID-19 in their 
departments. It was observed that $3.7 \%$ of the participants did not cover their ultrasound probe with disposable cellophane or protective gloves, as shown on Fig. 3.

Fig. 4 shows the participant response to questions on their operational protocols in their departments AFTER the outbreak of COVID-19. There are changes arising from the onset of COVIS-19, with increased use of face mask from $24.3 \%$ before to $89.7 \%$. The number of respondents who used hand gloves increased following the outbreak of COVID-19, from $75.7 \%$ to $93.5 \%$, as shown in Fig. 4. The difference is statistically significant $(\mathrm{p}<0.01)$.

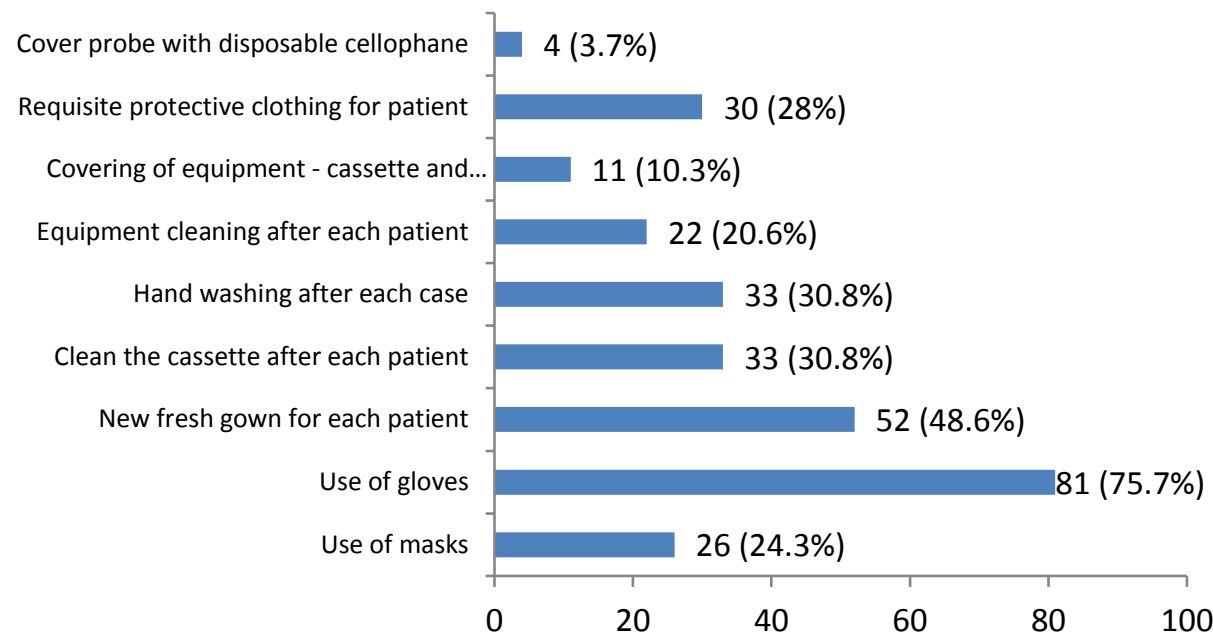

Fig. 3. Response to operational protocol before the outbreak of COVID-19.

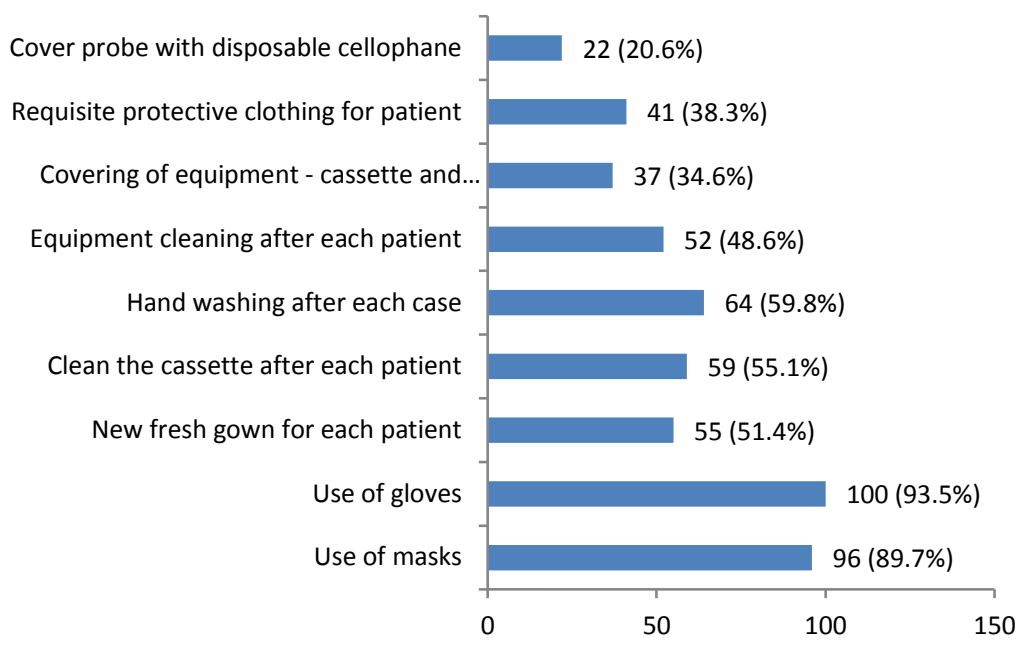

Fig. 4. Response to operational protocol After the outbreak of COVID-19.

Responses to the availability and use of personal protective equipment (PPE) by radiographers showed that $60(56.1 \%$ ) were not conversant with the right sequence for taking off PPE as shown in Fig. 5. Up to $75 \%$ of participants acknowledge the availability of hand sanitizers in imaging rooms, while $62.6 \%$ agree there is availability of running water in exposure rooms. Nearly $60 \%$ of respondents appear not to have protective face masks and other protective clothing, while a little over $50 \%$ use face masks and other protective clothing provided (Fig. 5).
Participants acknowledged the availability of disinfectants at their disposal $(79.4 \%)$. Over $60 \%$ confirm there were hand sanitizers at the entrances to the x-ray rooms. Materials like information leaflets, Help lines, Infectious (yellow) bins as well as ventilators were in very short supply (Fig. 6).

In Fig. 7, more than $60 \%$ of the participants confirmed that they did not practice deep cleaning of units, nor did they allow for a dry time of 20-30 minutes before letting in patients. Results also show that most respondents $(85.1 \%)$ were not provided with emergency $\mathrm{x}$-ray or ultrasound imaging units designated for COVID-19 cases. 


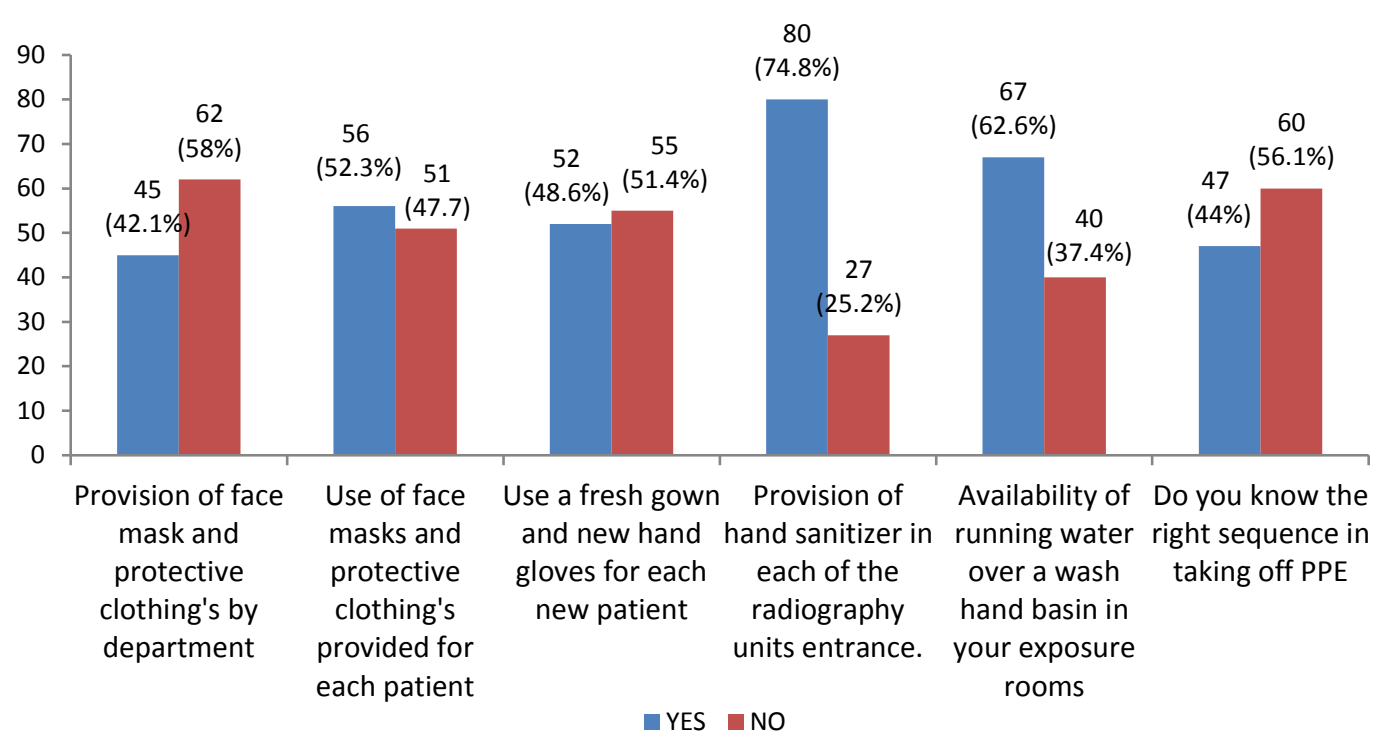

Fig. 5. Response to the availability and use of personal protective equipment (PPE) by radiographers.

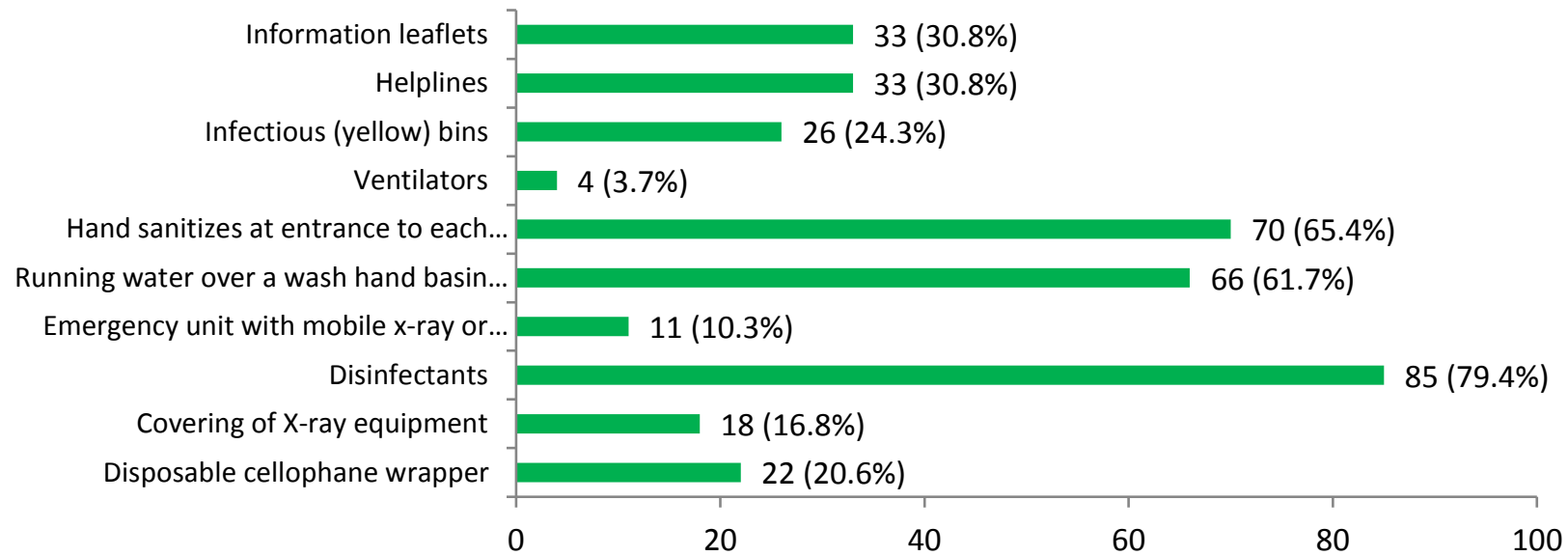

Fig. 6. Response to the availability of materials at radiographers disposal in the department after the outbreak of COVID-19.

Performing Deep cleaning of units and allowed for 20 - 30 min to dry before attending to a...

Provision of infectious bin (Yellow) for the disposal of your protective gears

Covering of ultrasound probe / transducer with protective gloves

Provision of emergency unit with mobile $x$-ray or ultrasound machines, for suspected COVID-..

Assisted by a radiographer colleague in handling the equipment and cassettes

Clearning and disinfecting Of X-ray Accessories

Covering the X-ray cassette and couch with disposable cellophane for each patient

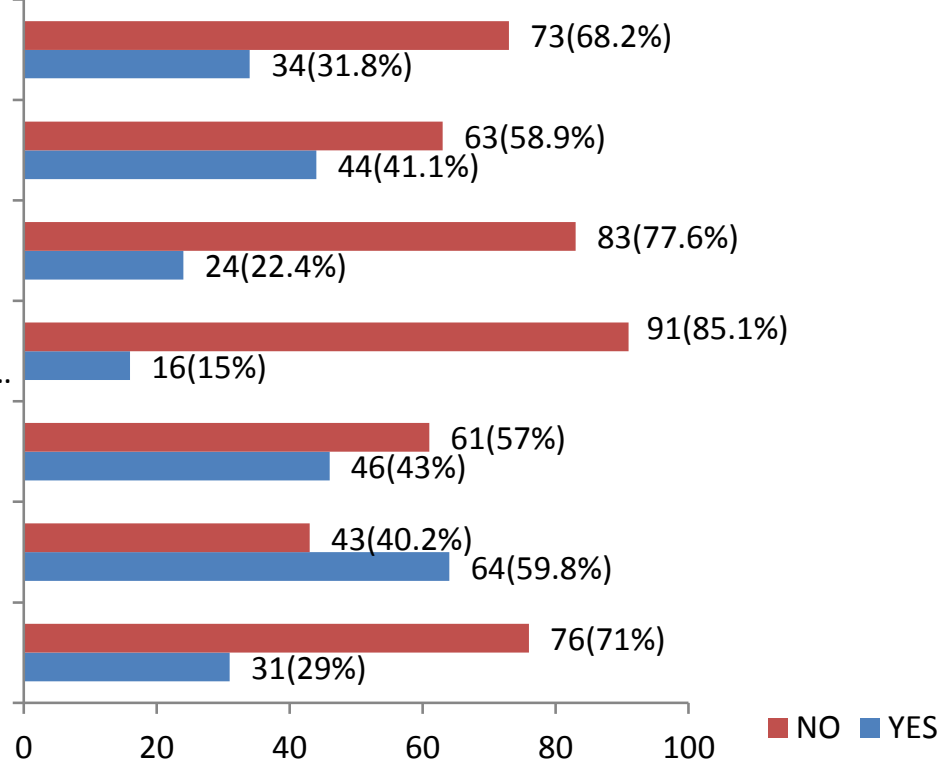

Fig. 7. Response to protection of radiographic equipment during COVID-19 outbreak. 


\section{Radiographic equipment}

Response to protection of radiographic equipment during COVID-19 outbreak before and after attending to patients in the departments.

Response to cleaning of accessories on USS, CT, and MRI machines during COVID-19 outbreak in the departments.

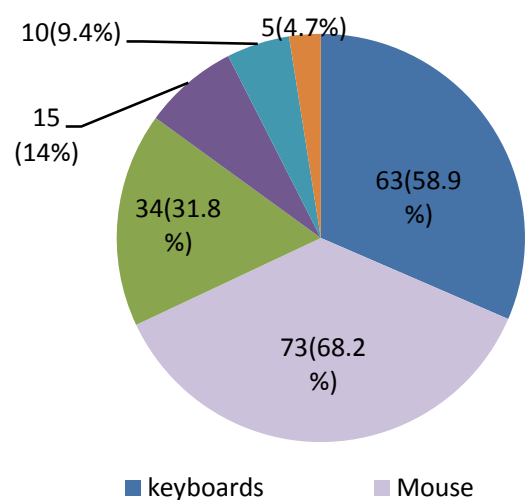

Fig. 8. Response to cleaning of accessories on USS, CT, and MRI machines.

\section{E. Emergency training}

Only $15 \%$ of respondents had undergone emergency training on issues pertaining to COVID-19 (Fig. 9 a, b). Of these, 9 were trained in limiting human to human transmission, while 6 were trained on patient care. While over $40 \%$ of respondents were aware of time restrictions with a single patient in the imaging room (Figure 10 a), nearly a quarter $(21 \%)$ still spent $>15$ minutes with patients in $X$-ray rooms (Fig. 10 b). Less than $10 \%$ of respondents spent over 15 minutes with patients in Ultrasound rooms, while $38.3 \%$ (40) spent $>15$ minutes with patients in CT rooms (Fig. $10 \mathrm{c}$ and d).

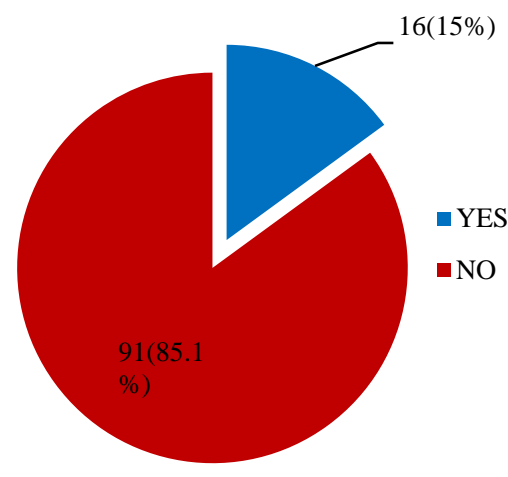

a) Have you had any training on emergency procedures during the COVID-19 outbreak.

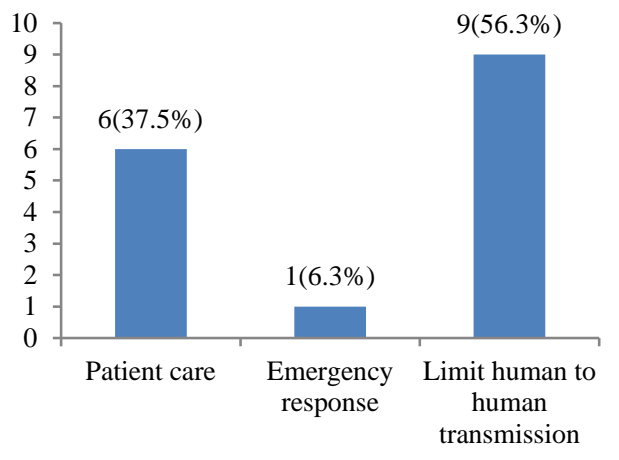

b) If you have had emergency training, what type.

Fig. 9. Response to emergency training during COVID-19 outbreak.

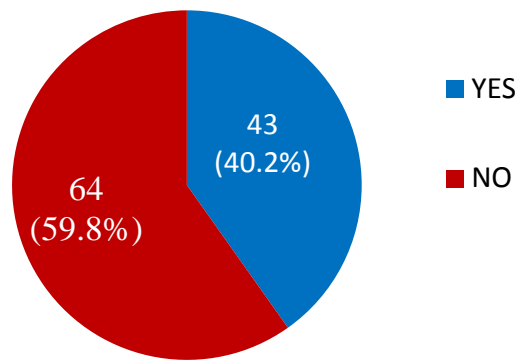

a) Are you aware that you are not to spend more than 15 minutes in the exposure, ultrasound, CT, and MRI scan room.

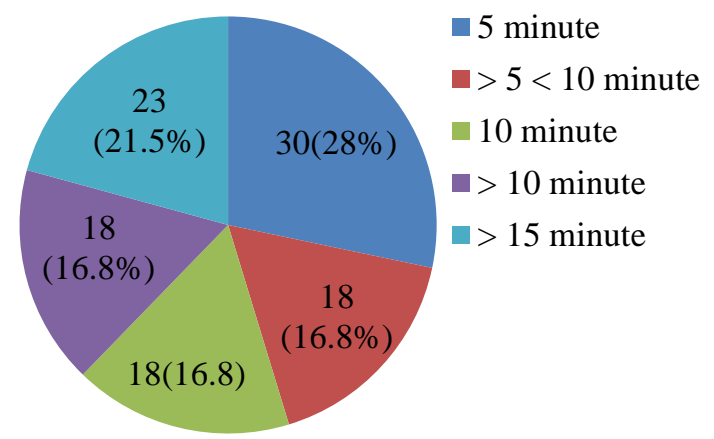

b) Approximate duration of time spent in X-ray exposure rooms.

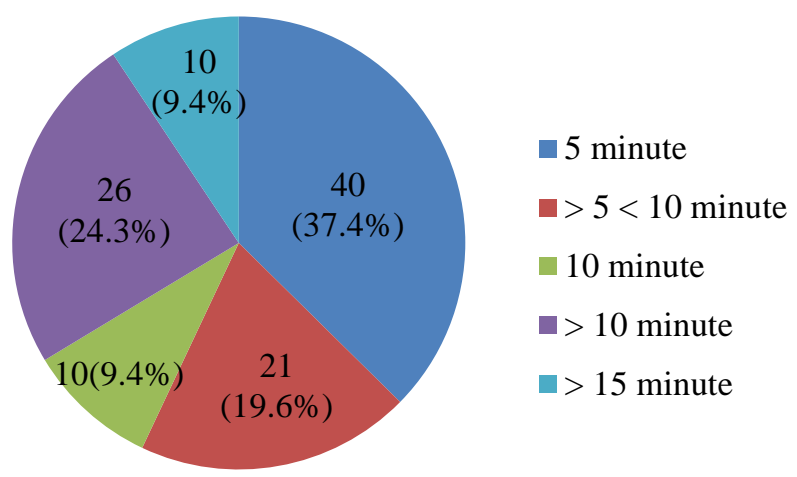

c) Approximate duration of time spent in USS scan rooms.

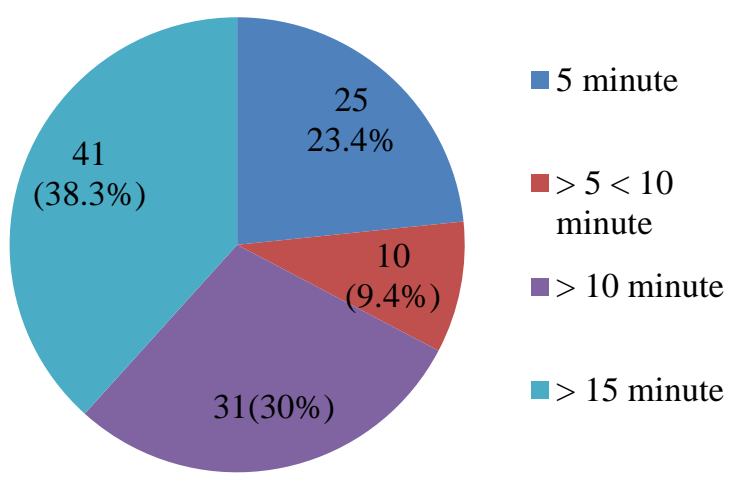

(d) Approximate duration of time spent in CT \& MRI scan rooms. Fig. 10. Response to duration of time spent while attending to patient during COVID-19 outbreak.

\section{F. Isolation centers}

Findings from the current study reveal that while a third of participants can point to a COVID-19 isolation Teams for their hospitals, over 50\% said there were no isolation centres in their facilities. While at least $41 \%$ of the participants claim 
there were isolation centres in their hospitals, there is evidence to show that no provision was made for imaging equipment in such centres $(82.2 \%)$ and there were no designated equipment anywhere, for imaging confirmed COVID-19 patients (Fig. 11). In addition, over $60 \%$ of respondents were not in the know of the Radiographer attached to the COVID-19 Team (Fig. 12).

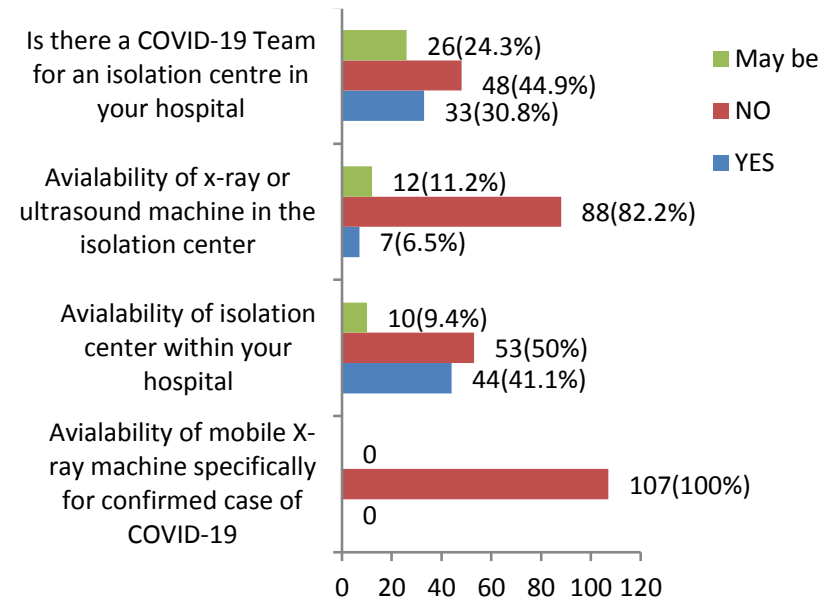

Fig. 11. Response to availability of isolation centers and equipment.

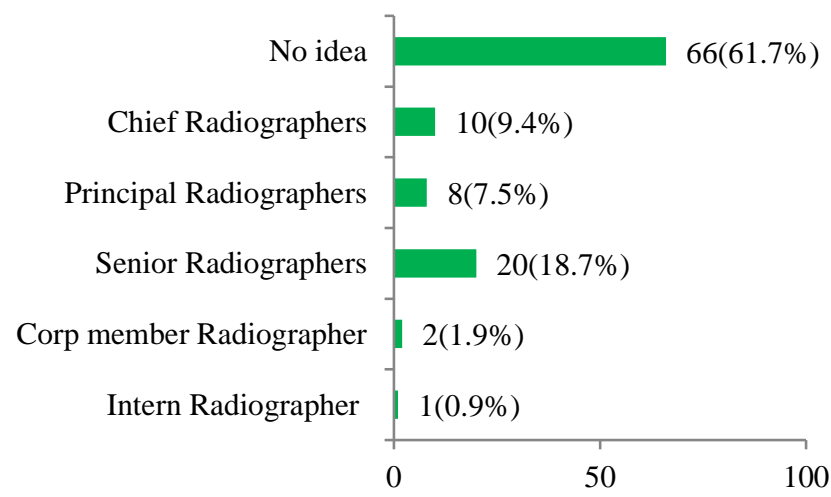

Fig. 12. Response to radiographers attached to the COVID-19 team for an isolation centre.

\section{DISCUSSION}

Information deducted from the current study shows that there have been changes in Departmental protocols or procedures following the onset of COVID-19 (Figure 3\&4), with an increase in the use of face masks from $24.3 \%$ before to $89.7 \%(\mathrm{p}<0.01)$, although there is a little difference in $(\mathrm{p}$ $<0.01)$ the use of gloves among radiographers before and after the COVID-19 outbreak $(75.7 \%$ and $93.5 \%$ respectively).This may be an indication of a response to the pandemic, rather than a proactive preparation for its advent. The absence of an appointment system and guidelines for prevention of person to person transmission suggests as much.

A little over half of the participants in the study $60(56.1 \%)$ of the respondents knew the right sequence for taking off PPEs. The WHO guidelines for reducing person to person infection and maintenance of personal hygiene [19] was largely followed in many facilities as indicated by over $60 \%$ of the participants ( $\mathrm{N}=67$ or $62.6 \%$ ). These alluded to having running water over a wash hand basin in the exposure rooms. Interestingly, hand washing after each examination increased with the advent of the COVID-19.

The use of fresh changing gowns and new hand gloves per patient was not practiced by the entire respondents. Only $48.6 \%$ of participants agreed undertook this practice as against $51.4 \%$ who did not. This practice may be hindered by availability of sufficient numbers of such gowns and hand gloves to necessitate regular changes for every patient. It is recommended that supplies of these materials are increased in this pandemic season.

Further observation of the responses show that the availability of some materials at radiographers' disposal in the department improved after the outbreak of COVID-19. Fig. 6, shows that $85(79.4 \%)$ were provided with disinfectants, $70(65.4 \%)$ were provided with hand sanitizers at the entrances to the radiography rooms. However, information leaflets, yellow bins, ventilators, emergency equipment, among others, were in short supply. These measures fall far short of expectations for handling a pandemic of this magnitude.

Equipment were not well protected (Figures $7 \& 8$ ). The consequences of this may be an increase in the vehicles for transmission of the disease. This can be avoided by simply wiping off equipment after each patient (although this may have time implications) or covering same with disposable covers during examinations.

One very crucial aspect of preparedness is training of personnel. Considering the novelty of the n-Cov 19 virus, investment in personnel training is crucial to successfully combating the disease. Radiographers in this study were largely unfamiliar with requirements for fighting the pandemic, except in patient care, person to person transmission and emergency response (Fig. 9). Training and re-training should be stepped up using available resources from WHO, NCDC through webinars and where possible departmental seminars.

Operator awareness of duration with a single patient in ray room is rather poor with up to a fifth of participants spending above 15 minutes in the $\mathrm{x}$-ray rooms. About a third of respondents spent over 15 minutes in CT and MRI rooms (Fig. 10). Increased training on emergency techniques would reverse this.

Perhaps the result indicated in Fig. 11 best describes the state of preparedness of the Healthcare systems in review. Only a third of respondents confirmed that there were COVID-19 teams set up in their facilities. Amazingly, just over $40 \%$ claim they had isolation centers in their hospitals, with more than $80 \%$ confirming the non-availability of any $\mathrm{x}$ ray or ultrasound equipment in existing Isolation centers. Where available, such Isolation centers were not equipped with dedicated $\mathrm{x}$-ray equipment for confirmed COVID-19 patients. In addition, most of the respondents (over 60\%) had no idea if there was a Radiographer on the COVID-19 teams in their centers.

The foregoing reflects a generally alarming situation especially when one considers the lack of sufficient testing centers. While we acknowledge the high cost of setting up Xray diagnostic facilities, the possibility of using it as a first line of management where the laboratory testing is unavailable makes it a necessary investment. This is made 
difficult by the apparent poor preparedness of medical imaging facilities, and perhaps, personnel, to receive COVID-19 cases.

\section{CONCLUSION}

This study has highlighted the level of preparedness and gaps in the radiography sector of the healthcare system in Nigeria. The radiography departments have made some effort at improving working procedures for radiographers after the outbreak of the COVID-19 pandemic. However, a lot of areas requires urgent attention. These include development of appointment systems, provision of guidelines to reduce human to human transmission, auditing for suspected COVID-19 cases, provision of dedicated imaging equipment for suspected and confirmed cases of COVID-19 in the departments and also in isolation centers, and the immediate setting of Isolation centers where there are none. Also, emergency training should be organized for all radiographers, some of whom should be made part of the COVID-19 team in all isolation centers.

\section{CONFLICT OF INTEREST DisCLOSURE}

The authors have no interest to disclose.

\section{LIMITATION}

Apart from the widely recognized limitation of questionnaire data [20], the respondent may be bias in their opinion and may have not responded sincerely to the questions. Also, there is a recognized variation that exist between the different radiography departments within the country.

\section{ACKNOWLEDGMENT}

The authors, wish to acknowledge the following senior radiographers:

- Mr Lucas Bassey Okon, Royal Berkshire hospital NHS foundation UK.

- Mr. Bright Ekakiettie, Royal Cornwall Hospital NHS foundation trust, Truro.

- Mr Aniekan Asuquo Jacob, University of Uyo teaching hospital, Uyo, Akwa Ibom state, Nigeria.

\section{REFERENCES}

[1] Biscayart C, Angeleri P, Lloveras S, Chaves TD, Schlagenhauf P, Rodríguez-Morales AJ. The next big threat to global health? 2019 novel coronavirus (2019-nCoV): What advice can we give to travellers?-Interim recommendations January 2020, from the LatinAmerican society for Travel Medicine (SLAMVI). Travel medicine and infectious disease. $2020 \mathrm{Jan} ; 33: 101567$.

[2] Ahmad T, Khan M, Khan FM, Hui J. Are we ready for the new fatal Coronavirus: scenario of Pakistan?. Human Vaccines \& Immunotherapeutics. 2020 Mar 3;16(3):736-8.

[3] Ahmad T, Khan M, Haroon TH, Nasir S, Hui J, Bonilla-Aldana DK, Rodriguez-Morales AJ. COVID-19: Zoonotic aspects. Travel Medicine and Infectious Disease. 2020 Feb 27.

[4] Huang C, Wang Y, Li X, Ren L, Zhao J, Hu Y, Zhang L, Fan G, Xu J, $\mathrm{Gu}$ X, Cheng Z. Clinical features of patients infected with 2019 novel coronavirus in Wuhan, China. The lancet. 2020 Feb 15;395(10223):497-506.

[5] Chan JF, Yuan S, Kok KH, To KK, Chu H, Yang J, Xing F, Liu J, Yip CC, Poon RW, Tsoi HW. A familial cluster of pneumonia associated with the 2019 novel coronavirus indicating person-to-person transmission: a study of a family cluster. The Lancet. 2020 Feb 15;395(10223):514-23
[6] Xu Z, Shi L, Wang Y, Zhang J, Huang L, Zhang C, Liu S, Zhao P, Liu $\mathrm{H}$, Zhu L, Tai Y. Pathological findings of COVID-19 associated with acute respiratory distress syndrome. The Lancet respiratory medicine. 2020 Apr 1;8(4):420-2.

[7] Template: COVID-19 pandemic data. Available at https://en.wikipedia.org/wiki/Template:COVID-19_pandemic_data [Accessed on 20/5/2020]

[8] COVIDE-19 case update by Nigeria Centre for Disease Control (NCDC) 2020. Available at https://covid19.ncdc.gov.ng/ [Accessed on 20/5/2020]

[9] Chandy PE, Nasir MU, Srinivasan S, Klass D, Nicolaou S, Babu SB Interventional radiology and COVID-19: evidence-based measures to limit transmission. Diagn Interv Radiol. 2020 Mar 31.

[10] Bastola A, Sah R, Rodriguez-Morales AJ, Lal BK, Jha R, Ojha HC, Shrestha B, Chu DK, Poon LL, Costello A, Morita K. The first 2019 novel coronavirus case in Nepal. The Lancet Infectious Diseases. 2020 Mar 1;20(3):279-80.

[11] Kim H. Outbreak of novel coronavirus (COVID-19): What is the role of radiologists?, (2020): 1-2.

[12] "Beijing orders 14-day quarantine for all returnees". BBC News. 15 February 2020. Available at https://www.bbc.com/news/world-asiachina-51509248 [Retrieved 21 April 2020].

[13] "Egypt announces first Coronavirus infection". Egypt Today. Available at https://www.egypttoday.com/Article/1/81641/Egypt-announcesfirst-Coronavirus-infection [Retrieved 21 April 2020]

[14] "FIRST CASE OF CORONA VIRUS DISEASE CONFIRMED IN NIGERIA". Nigeria Centre for Disease Control. 28 February 2020 Available at https://ncdc.gov.ng/news/227/first-case-of-corona-virusdisease-confirmed-in-nigeria [Retrieved 21 April 2020].

[15] Maclean, Ruth; Dahir, Abdi Latif (2020). "Nigeria Responds to First Coronavirus Case in Sub-Saharan Africa". The New York Times. Avialable https://www.nytimes.com/2020/02/28/world/africa/nigeriacoronavirus.html [Retrieved 21 April 2020].

[16] Hansell DM, Bankier AA, MacMahon H, McLoud TC, Muller NL, Remy J. Fleischner Society: glossary of terms for thoracic imaging. Radiology. 2008 Mar;246(3):697-722.

[17] Song F, Shi N, Shan F, Zhang Z, Shen J, Lu H, Ling Y, Jiang Y, Shi Y Emerging 2019 novel coronavirus (2019-nCoV) pneumonia. Radiology. 2020 Apr;295(1):210-7

[18] Chung M, Bernheim A, Mei X, Zhang N, Huang M, Zeng X, Cui J, Xu W, Yang Y, Fayad ZA, Jacobi A. CT imaging features of 2019 novel coronavirus (2019-nCoV). Radiology. 2020 Apr;295(1):202-7.

[19] World Health Organization. Rational use of personal protective equipment for coronavirus disease ( COVID-19) and considerations during severe shortages: interim guidance, 6 April 2020. World Health Organization; 2020.

[20] Burns R. introduction to research methods. London: SAGE; 2000

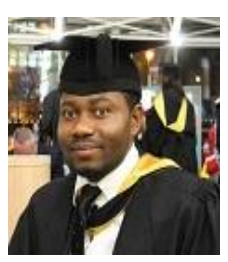

Mr. Akpaniwo G. M., graduated from the prestigious University of Calabar Nigeria, with B.Sc (Hons) Radiography. In 2010, he joined Usmanu Danfodiyo University teaching hospital Sokoto, where he rendered his services in General radiography, computed tomography (CT) and Magnetic resonance imaging(MRI). He attended the University of Bradford, UK in 2014 and obtain a Master's degree in Imaging in Medicine. He is a lecturer at the Radiography department of the Usmanu Danfodiyo University Sokoto, where he is providing his valuable services in the grooming of undergraduate radiographer students.

To quote some of his remarkable publications:

[1] Akpaniwo G. M., Sadiq A. A., Danfulani M., Abubakar U., Mohammed A., Iliyasu Y. I., Shehyi O. P., (2018). Challenges faced by Undergraduate Radiography students in Sokoto Northwest Nigeria, during long vacation clinical posting. International Journal of Health Science and Research.8(2): pp. 29 - 36. ISSN: 2249-9571.

[2] Akpaniwo G M., Boynes S., Danfulani M., Chigozie N I., Umar A., (2016). Should MRI replace mammography as the initial screening modality for asymptomatic women aged $18-45$ years at high risk of developing breast cancer? A systematic review. Asian Journal of Medical Sciences. 7(5): 1-9. e-ISSN:2091-0576, p-ISSN:2467-9100. DOI: 10.3126/ajms.v7i5.14743

[3] Akpaniwo G.M., Suleiman A.M., Nwobi I.C., Yunusa G.H., Sadiq A.A., Abubakar U., Mohammed A., Iliyasu Y.I., (2019). Patient X-Ray Entrance Surface Dose at a Tertiary Hospital in Sokoto, North-West Nigeria. Journal of Radiography \& Radiation Sciences. 33(1): pp. 1 - 5. ISSN: 11157976. 
Mr. Adebayo O.D., is a native of Oyo State, Nigeria. $\mathrm{He}$ was educated in Nigeria and the United Kingdom and holds the following qualifications; DIR (Radiography) (1993), Lagos Nigeria; BSc (Radiography) Queen Margaret University, Edinburgh UK (2007); PGCert (Computed Tomography) University of Bradford, Bradford UK (2010); PG Cert (CT Head Reporting) University of Bradford, UK (2016); MSc. Medical Imaging, University of Bradford, UK (2017). While in Nigeria he worked as a senior Radiographer at the National Hospital for Women and Children, Abuja, Nigeria. He is currently a senior Radiographer in the CT suite at the Department of Radiology and Imaging, Mercy University Hospital, Cork Republic of Ireland.

To quote some of his remarkable publications:

[1] Adebayo OD, Culpan G. Diagnostic accuracy of computed tomography perfusion in the prediction of haemorrhagic transformation and patient outcome in acute ischaemic stroke: A systematic review and metaanalysis. European stroke journal. 2020 Mar;5(1):4-16. DOI: $10.1177 / 2396987319883461$

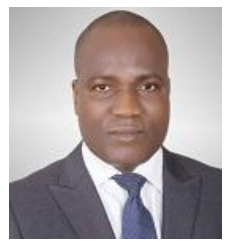

Prof. Mark Chukwudi Okeji graduated from the prestigious University of Nigeria, Nsukka with B.Sc (Hons) Radiography. He went ahead to obtain M.Sc and $\mathrm{PhD}$ in Radiography from the University of Nigeria, Nsukka. He was the first to obtain a PhD in Radiography from any department in Nigeria and indeed West Africa. Prior to joining University of Nigeria as a Lecturer in 2006, he had worked as a clinical Radiographer at National Orthopaedic Hospital, Enugu where he rose to the rank of Assistant Chief Radiographer. Before being appointed the Registrar/CEO of Radiographers Registration Board of Nigeria (RRBN), he was the; Associate Dean, Faculty of Health Sciences and Technology, University of Nigeria, Enugu Campus, - Senior Lecturer in Department of Radiography and Radiological Sciences, University of Nigeria, Enugu Campus, • Immediate Past President, The Association of Radiographers of Nigeria $(\mathrm{ARN})$,

To quote some of his remarkable publications:

[1] Okeji MC, Anakwue AM, Agwuna K. Radiation exposure from diagnostic radiography: an assessment of X-ray beam collimation practice in some Nigerian Hospitals. Internet Journal of Medical Update-EJOURNAL. 2010;5(2).

[2] Okeji MC, Agwu KK, Idigo FU. Natural radioactivity in cultivated land in the vicinity of a phosphate fertilizer plant in Nigeria. Radiation Physics and Chemistry. 2012 Dec 1;81(12):1823-6.

[3] Okeji MC, Anakwue AC, Agwuna KK, Chinweuba AC, Eze JC. Does preparatory information reduce anxiety and pain during hysterosalpingography procedure. Jokull. 2013;63(6):387-94.

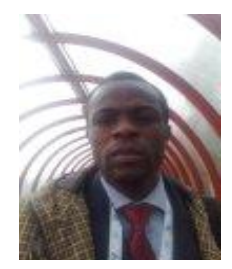

Prof. Nneoyi Egbe, is a Professor of Radiography \& Medical Physics and a lecturer at the University of Calabar. He hold degrees in Physics and Medical Physics from the prestigious University of Ibandan and Aberdeen, UK.

To quote some of his remarkable publications:

[1] Egbe NO, Heaton B, Sharp PF. A simple phantom study of the effects of dose reduction (by $\mathrm{kVp}$ increment) below current dose levels on $\mathrm{CR}$ chest image quality. Radiography. 2010 Nov 1;16(4):327-32.

[2] Egbe NO, Inyang SO, Ibeagwa OB, Chiaghanam NO. Pediatric radiography entrance doses for some routine procedures in three hospitals within eastern Nigeria. Journal of medical physics/Association of Medical Physicists of India. $2008 \mathrm{Jan} ; 33(1): 29$.

[3] Egbe NO, Heaton B, Sharp PF. Application of a simple phantom in assessing the effects of dose reduction on image quality in chest radiography. Radiography. 2010 May 1;16(2):108-14. 